\title{
Individual Risk Factors of the Metabolic Syndrome in Adult Patients with Growth Hormone Deficiency - A Cross-sectional Case-control Study
}

Authors

Affiliations

\author{
I. Uzunova ${ }^{1}$, G. Kirilov ${ }^{1}$, S. Zacharieva ${ }^{1}$, A. Shinkov ${ }^{1}$, A.-M. Borissova ${ }^{1}$, K. Kalinov ${ }^{2}$
}

Clinical Centre of Endocrinology, Medical University of Sofia, Sofia, Bulgaria

${ }^{2}$ New Bulgarian University, Sofia, Bulgaria

\author{
Key words \\ - growth hormone deficiency \\ - metabolic syndrome \\ - abdominal obesity \\ - dyslipidemia \\ - cardiovascular risk
}

\begin{abstract}
$\nabla$

Objective: Growth hormone deficiency in adults (GHDA) is considered to be associated with increased cardiovascular risk, most commonly reflected by the prevalence of the metabolic syndrome (MS). However, there are still a limited number of studies comparing directly the MS prevalence in GHD patients to that in general population. The aim of this study was to investigate the individual risk factors of the MS in a cohort of GHD patients and to compare its prevalence with an age- and sex-matched control group.
\end{abstract}

Design: A cross-sectional case-control study.

Methods: In total, 54 adult patients with GHD (childhood onset GHD (COGHD): $n=19$, adult onset GHD (AOGHD): $n=35$ ) and 2153 control subjects were studied. GHD was diagnosed according to the Endocrine Society Clinical Practice Guideline recommendations from 2011 and MS was scored by the NCEP-ATP III definition.

\section{Introduction \\ Introduction}

first decision 03.09.2014

accepted $\quad 17.09 .2014$

Bibliography

DOI http://dx.doi.org/

10.1055/s-0034-1390460

Published online:

November 20, 2014

Exp Clin Endocrinol Diabetes

2015; 123: 39-43

(c) J. A. Barth Verlag in

Georg Thieme Verlag KG

Stuttgart · New York

ISSN 0947-7349

Correspondence

I. Uzunova

2 Zdrave Str.

1431 Sofia

Bulgaria

dr_uzunova@yahoo.com
Growth hormone deficiency in adults (GHDA) results in the lack of the lipolytic and anabolic effects of growth hormone (GH), which causes: substantial changes in body composition, an atherogenic lipid profile and subsequent insulin resistance with compensatory hyperinsulinemia [1-5]. These data demonstrate the association between the metabolic syndrome (MS) and GHDA and emphasize the social significance of hyposomatotropism as a disease accompanied with an adverse cardiovascular risk profile.

A couple of large retrospective and prospective studies reported an increased risk of premature death among patients with hypopituitarism, due predominantly to the higher prevalence of cardio- and cerebrovascular diseases [6-10]. Some authors hypothesized that this could have been a
Results: The main metabolic abnormalities in GHD group were increased waist circumference (50.0\%), low HDL-cholesterol (42.6\%) and hypertriglyceridemia (40.7\%) and their prevalence was significantly higher $(\mathrm{p}=0.013, \mathrm{p}=0.019$ and $\mathrm{p}=0.010$, respectively) than in control group, where increased blood pressure prevailed $(64.2 \%, p<0.0001)$. However, the difference in the MS prevalence between the 2 groups (29.6\% vs. $24.9 \%$ in controls) failed to reach statistical significance $(p=0.429)$. Patients with MS from both groups did not differ significantly in their metabolic profile (except for increased blood pressure), mean age and gender distribution.

Conclusions: Although GHDA was associated with the development of visceral obesity and dyslipidemia, these adverse cardiovascular risk factors did not determine a higher prevalence of the MS in Bulgarian GHD patients compared to control subjects. Furthermore, the individual risk factors of the MS did not significantly differ between patients with MS from both groups.

consequence of untreated GHD [6,11]. Although such trials were usually hampered by the small number of patients or the limited study period, these findings influenced considerably the more frequent use of GH replacement therapy in adult patients with hyposomatotropism, aiming to improve quality of life and to prevent the longterm complications of GHD.

During these years the number of treated patients has become large enough to enable the conduction of studies of both the short- and the longterm effects of GH substitution. Despite the initial encouraging results [12-16] some controversial data regarding cardiovascular risk have recently been obtained [17-22]. In the absence of unequivocal mortality evidence for the benefit of $\mathrm{GH}$ replacement, heavy reliance has been placed on changes in surrogate markers of vascular disease. This accentuates the necessity to critically 
evaluate the factors that contribute to the cardiovascular risk in adult patients with GHD.

Based on the fact that the overall cardiovascular risk profile is most commonly reflected by the prevalence of the MS, the aim of this study was to investigate the individual risk factors of the MS in a cohort of GHD patients and to compare its prevalence with an age- and sex-matched control group.

\section{Material and Methods \\ $\nabla$}

\section{Patients}

In total, 54 patients ( 20 males and 34 females) aged $20-80$ years (20-60 years for males, 20-80 years for females) were included in the analysis. They were diagnosed with hyposomatotropism in the Clinical Centre of Endocrinology, Sofia, from October 2011 to February 2013. Medical history of acromegaly was used as exclusion criterion. Adult onset patients (AOGHD) accounted for $64.8 \%(n=35)$ and childhood onset (COGHD) - for the remaining $35.2 \%(n=19)$. Major part of the patients was diagnosed with severe GHD - levels of GH and IGF-1 below the analytical sensitivity of the assays were found in $75.9 \%(n=41)$ and $53.7 \%$ $(\mathrm{n}=29)$ of the subjects, respectively.

At study entry all concomitant pituitary deficiencies were adequately substituted. Patients were treated with hydrocortisone, l-thyroxine, testosterone or estrogen in combination with prostagens in premenopausal women only. Substitution therapy dosages were as required for normalization of clinical and biochemical (mean fT4 level: $14.15 \pm 2.67 \mathrm{pmol} / \mathrm{l}$; mean testosterone level: $18.65 \pm 8.38 \mathrm{nmol} / 1)$ parameters of pituitary deficiencies. None of the patients had received GH substitution during the last 2 years prior to enrollment in the study. Subjects who had undergone GH replacement therapy in the past were found only among COGHD patients - 73.7\% $(n=14)$ of this subgroup. Antihypertensive and lipid-lowering medication was initiated by the treating physician as follows: $25.9 \%(n=14)$ of the patients received antihypertensive drugs and $11.1 \%(n=6)$ were treated with lipid-lowering medication.

The investigation was approved by the local ethical committee and informed consent has been obtained from each patient after full explanation of the purpose and nature of all procedures used.

\section{Control subjects}

Control subjects were recruited from a population-based study of thyroid diseases and diabetes conducted in Bulgaria. It included 2153 subjects (977 males and 1176 females), aged 20-94 years (20-91 years for the males and 20-94 years for the females).

\section{Diagnostic protocol}

The following study parameters were assessed: waist circumference, systolic and diastolic blood pressure, fasting plasma glucose, HDL-cholesterol and triglycerides. All blood samples were taken after 8-12 h overnight fast.

Serum GH concentrations were determined by a solid-phase 2-site immunoradiometric assay (Immunotech, Beckman Coulter Co., Prague, Czech Republic). The sensitivity of this assay was $<0.1 \mathrm{mIU} \mathrm{l}^{-1}$. The intra- and inter-assay coefficients of variation were $1.5 \%$ and $14.0 \%$, respectively. Extremely low cross reactivities were obtained against several related molecules (hCG, HPL, hpGH, prolactin). Serum IGF1 was measured with an immunoradiometric kit after acid-alcohol extraction (Immunotech; Beckman Coulter Co., France). Analytical sensitivity was $<0.26 \mathrm{nmol} \mathrm{l}^{-1}$. The intra- and inter-assay coefficients of variation were $6.3 \%$ and $6.8 \%$, respectively.

GHD was diagnosed according to the Endocrine Society Clinical Practice Guideline recommendations from 2011 [23]: 1. In the presence of deficiencies of 3 or more pituitary axes the low ageadjusted serum IGF1 level was considered sufficient to prove GHD. 2. In all other cases the insulin tolerance test was performed and peak GH level $<5.1 \mu \mathrm{g} \mathrm{l^{-1 }}$ was established as a cutoff value.

MS was scored by the National Cholesterol Education Program - Adult Treatment Panel III (NCEP-ATP III) criteria [24].

\section{Statistical analysis}

Statistical calculations were performed using SPSS for Windows, version 13.0. The age of the subjects was expressed as Mean \pm SD. Independent Samples Test was used for the comparison of the mean age between groups and Pearson Chi-Square test was employed for comparing age distribution between the 2 studied groups. Categorical data (sex, presence of the MS and its components) were analysed by Fisher's Exact Test. The probability value used to identify significance was $\mathrm{P}<0.05$ in all cases.

\section{Results}

$\nabla$

The individual risk factors of the MS were evaluated in 54 patients (20-80 years, mean age $45.4 \pm 15.7$ years) with GHD (group A) and in a control group of 2153 subjects (20-94 years, mean age $47.2 \pm 14.5$ years), participating in a Bulgarian population-based study (group B). No significant difference in age and gender between both cohorts was found $(\mathrm{p}=0.365$ and $\mathrm{p}=0.268$, respectively) ( 0 Table $\mathbf{1}$ ).

The underlying causes of hyposomatotropism included: hypothalamic-pituitary tumors (non-functioning adenoma $-\mathrm{n}=20$, prolactinoma $-n=3$, Cushing's disease $-n=1$, and craniopharyngioma $-n=9$ ) with or without surgery (transsphenoidal $n=27$, transcranial $-n=13)$ and radiotherapy $(n=7)$, congenital multiple pituitary hormone deficiency $(n=11)$, isolated idiopathic GHD $(n=5)$, and Sheehan's syndrome $(n=4) .42$ subjects (77.8\%) had developed GHD and 3 other pituitary hormone deficiencies, 3 patients (5.5\%) showed deficiencies of 2 other pituitary axes, 4 patients $(7.4 \%)$ - one other hormone deficiency and the remaining 5 subjects $(9.3 \%$ ) had isolated GHD. The prevalence of the concomitant anterior pituitary deficiencies was practically identical: hypogonadism and hypothyroidism were

Table 1 Characteristics of the patients with growth hormone deficiency (GHD) and the control subjects.

\begin{tabular}{|lll|}
\hline Parameter & $\begin{array}{l}\text { Patients with GHD } \\
\text { (group A) }\end{array}$ & $\begin{array}{l}\text { Control group } \\
\text { (group B) }\end{array}$ \\
\hline Number of subjects & 54 & 2153 \\
\hline Males/Females (\%) & $37.0 / 63.0$ & $45.4 / 54.6\left(\mathrm{p}-\mathrm{NS}^{*}\right)$ \\
\hline Age min-max (years) & $20-80$ & $20-94$ \\
\hline Mean age (years) & $45.4 \pm 15.7$ & $47.2 \pm 14.5\left(\mathrm{p}-\mathrm{NS}^{*}\right)$ \\
\hline$\leq 44$ years (\%) & 46.3 & $46.2\left(\mathrm{p}-\mathrm{NS}^{*}\right)$ \\
\hline 45-59 years (\%) & 40.7 & $34.6\left(\mathrm{p}-\mathrm{NS}^{*}\right)$ \\
\hline$\geq 60$ years (\%) & 13.0 & $19.2\left(\mathrm{p}-\mathrm{NS}^{*}\right)$ \\
\hline${ }^{*}$ - NS: $\mathrm{p}$-value is non-significant & \\
\hline
\end{tabular}


present in 46 of the subjects (85.2\%) and 44 of the patients (81.5\%) had hypocorticism.

The most prevalent metabolic abnormalities in the GHD group were increased waist circumference (50.0\%), low HDL-cholesterol (42.6\%) and hypertriglyceridemia (40.7\%). Meanwhile, in the control group high blood pressure (64.2\%) prevailed over increased waist circumference $(33.2 \%)$ and dyslipidemia (low HDL-cholesterol - 27.1\%; hypertriglyceridemia - 24.4\%).

Comparing the prevalence of each MS component between the subjects in both groups, significant difference was observed for all of the components, except for the increased levels of fasting glucose $(p=0.829)$. Abdominal obesity $(p=0.013)$, low HDL-cholesterol $(p=0.019)$ and hypertriglyceridemia $(p=0.010)$ were more prevalent in the GHD group. Only the prevalence of increased blood pressure ( $>130 / 85 \mathrm{mmHg}$ ) was more expressed in the control group $(\mathrm{p}<0.0001)$ ( $\odot$ Fig. 1$)$.

However, the difference in the MS prevalence among GHD patients and control subjects failed to reach statistical significance $(p=0.429)$. In group A the MS was present in $29.6 \%(n=16)$ of the patients. After dividing the subjects into 2 subgroups in accordance with the onset of the GHD - COGHD and AOGHD, a significant difference in the MS prevalence was found $(p=0.03)$. Among the 19 patients with COGHD only $10.5 \%(\mathrm{n}=2)$ fulfilled the criteria for MS, whereas among those with AOGHD $(n=35)$ the prevalence of the MS was $40.0 \%(n=14)$. In group B $24.9 \%$ of the subjects $(\mathrm{n}=537)$ were diagnosed with MS.

Analysing only those patients from both groups diagnosed with MS (in GHD group: $n=16$; in control group: $n=537$ ), we found no significant difference as far as mean age (GHD group $52.3 \pm 13.5$ years, control group $-52.7 \pm 13.7$ years; $p=0.919$ ) and gender distribution (males:females with MS in GHD group $50 \%: 50 \%$, in control group - $49.7 \%: 50.3 \% ; p=1.000$ ) were concerned. In GHD group the MS was characterized mainly with increased waist circumference (93.8\%) and dyslipidemia (hypertriglyceridemia - 87.50\%, low HDL-cholesterol - 81.3\%). In comparison, in control subjects with MS the most common metabolic abnormalities were high blood pressure (94.6\%) followed by obesity (82.4\%) and impairment of the lipid profile (hypertriglyceridemia $-69.2 \%$ and low HDL-cholesterol - 60.9\%). Furthermore, increased blood pressure was the only component which prevalence remained significantly different between the patients with MS in both groups $(p=0.002)$. Lipid abnormalities remained more prevalent in the patients with MS from the GHD group but this difference failed to reach statistical significance ( $\boldsymbol{\theta}$ Fig. 2 ).

\section{Discussion}

$\nabla$

In the present study we evaluated the individual risk factors of the MS in 2 age- and sex-matched cohorts - patients with GHD (group A) and a control group (group B) consisting of the participants in Bulgarian population-based study. The most common components of the MS in group A were increased waist circumference and dyslipidemia vs. increased blood pressure in group B. The incidence of the individual risk factors of the MS was relatively similar to the one found in other European cohorts of GHD patients [18-20]. As far as the control group was concerned, despite seeming rather high, the prevalence of increased blood pressure $(64.2 \%)$ was not surprising since a similar rate was reported in a Dutch study with a comparable prevalence of the MS in the general population [20]. The lower blood pressure

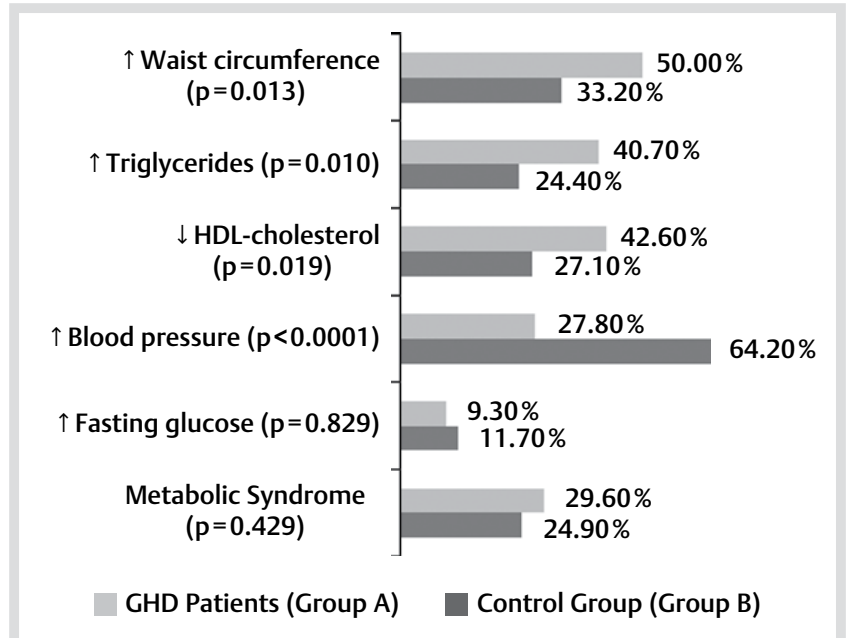

Fig. 1 Prevalence of the MS components in both groups.

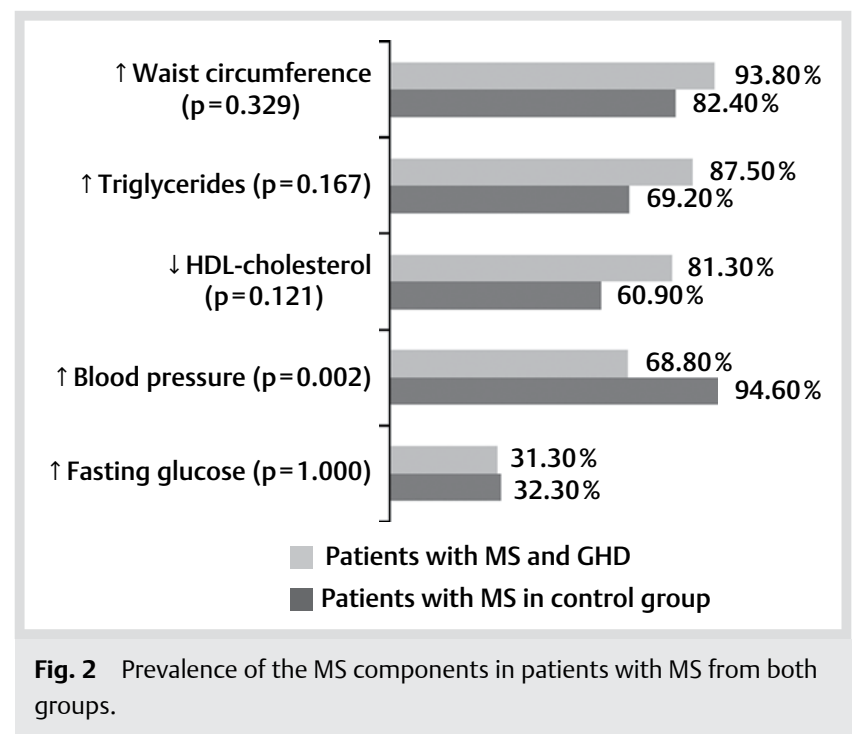

values in GHD patients were most likely related to the concomitant secondary adrenocortical insufficiency.

When comparing the individual risk factors between the 2 groups, some differences were demonstrated, namely the pronounced proneness to lipid abnormalities in GHD patients. That proves the hypothesis that the fundamental pathophysiological basis of the metabolic abnormalities in hyposomatotropism is the lack of the lipolytic effect of GH [25], resulting in visceral obesity. However, the difference in the MS prevalence among GHD patients and control subjects failed to reach statistical significance.

Moreover, the patients with MS from both groups did not differ significantly in their metabolic profile, regarding the fact that the prevalence of the MS components (except for increased blood pressure) was comparable. Analysing the gender characteristics of these subjects, we found that the MS was equally prevalent in men and women in both subgroups - a result consistent with the one reported by Claessen et al. [17] and Verhelst et al. [26]. Furthermore, the mean age of the patients with MS in both groups was comparable. These findings raise the question whether the unequivocal metabolic abnormalities in GHD patients actually result in the development of more severe MS 
and whether the latter occurs earlier in patients with hyposomatotropism than in general population.

Over the past 2 decades a number of studies of the cardiovascular risk factors associated with GHDA have been published $[1,2,4,27,28]$. The largest database, including more than 15000 GHD patients from 31 countries, is the international epidemiological study KIMS (Pfizer International Metabolic Database). Analysing these data, Abs et al. [1] proved that hyposomatotropism affected predominantly the lipid profile, waist circumference and body mass index. The results from this large cohort of patients, which were consistent with our data, confirmed that GHD was associated with the MS and therefore determined increased cardiovascular risk.

However, there are still a limited number of studies comparing directly the MS prevalence in GHD patients to that in general population [18-20].

Van der Klaauw et al. [18] demonstrated that the MS prevalence in patients with GHDA was twice higher than that in general Dutch population (38.0\% vs. $15.7 \%$ ). The discrepancy between these results and ours could possibly be explained by the much lower prevalence of the MS in Dutch control group and by the different characteristics of the patients. In our study approximately $1 / 3$ of them were with COGHD. As demonstrated in a number of publications $[3,29,30]$, the prevalence of the MS in COGHD is far lower than in AOGHD - approximately $10 \%$, in our study $-10.5 \%$. If we had considered the MS prevalence $(40.0 \%)$ only in those of our patients who were with AOGHD, the results would have been comparable. 2 other recent studies by Verhelst and al. [26] and by Claessen et al. [20] also reported a similar prevalence of the MS ( $43.1 \%$ and $41.0 \%$ respectively) in AOGHD patients (in the Dutch study less than $4 \%$ of the subjects were with COGHD).

Attanasio et al. [19] evaluated the prevalence of the MS in 2531 COGHD and AOGHD patients from Europe, USA and Canada. As far as European patients were concerned, the investigators were not able to find any suitable population for a direct comparison. Therefore the MS prevalence was retrieved from recent epidemiological studies in different countries and ranged from $17 \%$ (Spain) to 27\% (Germany). Relying on these data, it was assumed that the incidence of the MS in the European GHD cohort (35.6\%) was higher than that in the general population. As the authors mentioned, however, the frequency of the MS was not assessed in a representative sample of adults with GHD, but in a group of patients selected for initiation of GH substitution, thus creating a clear selection bias (it could not be ruled out that a number of patients with different risk for developing MS were excluded from the analysis).

Generally, when comparing results from various studies some other inevitable differences should be taken into account as well: the underlying causes of hyposomatotropism; the prevalence of other pituitary deficiencies; their adequate substitution; the proportion of patients who had undergone radiotherapy. A potential limitation of our study was that, due to its cross-sectional design, it was not possible to correct some of the potential contributors to the MS, such as: diet, physical activity, alcohol intake, smoking, social and economic status, family history. Firm conclusions on whether the MS was due to GHD per se or to any pre-existing metabolic abnormalities could therefore not be drawn from present results.

Practically, all the data known so far synonymously show that adults with GHD have an adverse cardiovascular risk profile which is likely to explain the increased cardiovascular mortality observed in patients with hypopituitarism. The beneficial effects of GH therapy on cardiovascular risk factors have been wellestablished in the short term but during the last decade a number of studies have demonstrated certain controversy as far as long-term effects are concerned [17,20-22]. Available literature on the exact net beneficial effect of GH replacement on overall cardiovascular risk and mortality rate shows inconsistent results. This accentuates the need for extended prospective studies following up larger cohorts of non-treated GHD patients and evaluating their cardiovascular risk profile in the long term compared to the one in substituted patients and in general population, as well as further risk-benefit and cost-effectiveness analyses.

In conclusion, data from our study proved that GHDA is associated with the development of visceral obesity and dyslipidemia. Nevertheless, these adverse cardiovascular risk factors did not determine a higher prevalence of the MS in Bulgarian GHD patients compared to control subjects. Furthermore, the individual risk factors of the MS did not significantly differ between patients with MS in the control group and in the GHD group. These results, as well as recent controversial data on cardiovascular risk and mortality from prospective studies of GH therapy long-term effects, emphasize the necessity to consider strictly the indications for initiating GH substitution in daily clinical practice and to evaluate individually in each patient whether prolonged, potentially life-long, and expensive replacement treatment is advisable.

\section{Acknowledgements}

$\nabla$

This work was supported by a research grant from the Medical University of Sofia (grant number 56/2011).

Declaration of interest: The authors declare that there is no conflict of interest that could be perceived as prejudicing the impartiality of the research reported.

\section{References}

1 Abs R, Feldt-Rasmussen U, Mattsson AF et al. Determinants of cardiovascular risk in 2589 hypopituitary GH-deficient adults - a KIMS database analysis. Eur J Endocrinol 2006; 155: 79-90

2 Verhelst J, Abs R. Cardiovascular risk factors in hypopituitary GHdeficient adults. Eur J Endocrinol 2009; 161: 41-49

$3 \mathrm{Lim} \mathrm{HH}$, Kang MJ, Yun IS et al. Prevalence and risk factors of the metabolic syndrome in young adults with childhood-onset hypopituitary growth hormone deficiency. Korean J Pediatr 2010; 53: 892-897

4 Gazzaruso C, Gola M, Karamouzis I et al. Cardiovascular risk in adult patients with growth hormone $(\mathrm{GH})$ deficiency and following substitution with GH - an update. J Clin Endocrinol Metab 2014; 99: 18-29

5 Johansson JO, Fowelin J, Landin $K$ et al. Growth hormone-deficient adults are insulin-resistant. Metabolism 1995; 44: 1126-1129

6 Rosén T, Bengtsson B-Å. Premature mortality due to cardiovascular disease in hypopituitarism. Lancet 1990; 336: 285-288

7 Bates AS, Van't Hoff W, Jones PJ et al. The effect of hypopituitarism on life expectancy. J Clin Endocrinol Metab 1996; 81: 1169-1172

8 Bülow B, Hagmar L, Mikoczy Z et al. Increased cerebrovascular mortality in patients with hypopituitarism. J Clin Endocrinol Metab 1997; 46: $75-81$

9 Svensson J, Bengtsson $B-A ̊$, Rosen $T$ et al. Malignant disease and cardiovascular morbidity in hypopituitary adults with or without growth hormone replacement therapy. J Clin Endocrinol Metab 2004; 89: 3306-3312

10 Tomlinson JW, Holden N, Hills RK et al. Association between premature mortality and hypopituitarism. West Midland Prospective Hypopituitarism Group. Lancet 2001; 357: 425-431 
11 Bengtsson $B$ - $A$. Untreated growth hormone deficiency explains premature mortality in patients with hypopituitarism. Growth Horm IGF Res 1998; 8: 480-485

12 al-Shoumer KA, Gray R, Anyaoku V et al. Effects of four years' treatment with biosynthetic human growth hormone $(\mathrm{GH})$ on glucose homeostasis, insulin secretion and lipid metabolism in GH-deficient adults. Clin Endocrinol (Oxf) 1998; 48: 795-802

13 Gibney J, Wallace JD, Spinks T et al. The effects of 10 years of recombinant human growth hormone $(\mathrm{GH})$ in adult $\mathrm{GH}$-deficient patients. J Clin Endocrinol Metab 1999; 84: 2596-2602

14 Gillberg P, Bramnert $M$, Thoren $M$ et al. Commencing growth hormone replacement in adults with a fixed low dose. Effects on serum lipoproteins, glucose metabolism, body composition, and cardiovascular function. Growth Horm IGF Res 2001; 11: 273-281

15 Götherström G, Svensson J, Koranyi J et al. A prospective study of 5 years of GH replacement therapy in GH-deficient adults: sustained effects on body composition, bone mass, and metabolic indices. J Clin Endocrinol Metab 2001; 86: 4657-4665

16 Svensson J, Fowelin J, Landin $\mathrm{K}$ et al. Effects of seven years of $\mathrm{GH}-$ replacement therapy on insulin sensitivity in GH-deficient adults. J Clin Endocrinol Metab 2002; 87: 2121-2127

17 Claessen KM, Appelman-Dijkstra NM, Adoptie DM et al. Metabolic profile in growth hormone-deficient (GHD) adults after long-term recombinant human growth hormone $(\mathrm{rhGH})$ therapy. J Clin Endocrinol Metab 2013; 98: 352-361

18 van der Klaauw A, Biermasz N, Feskens $E$ et al. The prevalence of the metabolic syndrome is increased in patients with GH deficiency, irrespective of long-term substitution with recombinant human GH. Eur J Endocrinol 2007; 156: 455-462

19 Attanasio AF, Mo D, Erfurth EM et al. Prevalence of metabolic syndrome in adult hypopituitary growth hormone $(\mathrm{GH})$-deficient patients before and after GH replacement. J Clin Endocrinol Metab 2010; 95: 74-81

20 Claessen KM, Appelman N, Pereira AM et al. Abnormal metabolic phenotype in middle-aged growth hormone deficient (GHD) adults despite long-term recombinant human $\mathrm{GH}$ (rhGH) replacement. Eur J Endocrinol 2013; 170: 265-274

21 Burger AG, Monson JP, Colao AM et al. Cardiovascular risk in patients with growth hormone deficiency: effects of growth hormone substitution. Endocr Pract 2006; 12: 682-689
22 van Bunderen CC, van Nieuwpoort IC, Arwert LI et al. Does growth hormone replacement therapy reduce mortality in adults with growth hormone deficiency? Data from the Dutch National Registry of Growth Hormone Treatment in adults. J Clin Endocrinol Metab 2011; 96: 3151-3159

23 Molitch ME, Clemmons DR, Malozowski S et al. Endocrine Society. Evaluation and treatment of adult growth hormone deficiency: an Endocrine Society clinical practice guideline. J Clin Endocrinol Metab 2011; 96: 1587-1609

24 Executive Summary of The Third Report of The National Cholesterol Education Program (NCEP). Expert Panel on Detection, Evaluation, And Treatment of High Blood Cholesterol In Adults (Adult Treatment Panel III). JAMA 2001; 285: 2486-2497

25 Salomon $F$, Cuneo RC, Hesp R et al. The effects of treatment with recombinant human growth hormone on body composition and metabolism in adults with growth hormone deficiency. N Engl J Med 1989; 321: 1797-1803

26 Verhelst J, Mattsson AF, Luger A et al. Prevalence and characteristics of the metabolic syndrome in 2479 hypopituitary patients with adultonset GH deficiency before GH replacement: a KIMS analysis. Eur J Endocrinol 2011; 165: 881-889

27 Murray RD, Wieringa G, Lawrance JA et al. Partial growth hormone deficiency is associated with an adverse cardiovascular risk profile and increased carotid intima-medial thickness. Clin Endocrinol (Oxf) 2010; 73: 508-515

28 Schneider HJ, Klotsche J, Wittchen HU et al. Effects of growth hormone replacement within the KIMS survey on estimated cardiovascular risk and predictors of risk reduction in patients with growth hormone deficiency. Clin Endocrinol (Oxf) 2011; 75: 825-830

29 Attanasio AF. Childhood onset adult growth hormone deficiency and hypopituitarism: a black box. In: Webb S, Chanson P. A decade of HypoCCS: the changing face of pituitary disease. Bristol, UK: Bioscentifica Ltd, 2008; 61-71

30 Attanasio AF, Lamberts SW, Matranga AM et al. Adult growth hormone (GH)-deficient patients demonstrate heterogeneity between childhood onset and adult onset before and during human GH treatment. J Clin Endocrinol Metab 1997; 82: 82-188 